\title{
Message from the Editor
}

It is with great pleasure and delight that I have assumed the role as Editor of the Canadian Journal of Neurological Sciences. The Journal has had strong leadership through its founding Editor Dr. R. Ross followed by the guidance of Dr. Robert G. Lee and more recently Dr. James Sharpe. We are all indebted to these editors and the editorial staff at the Journal for creating a self sufficient, strong and internationally recognized Journal. Unlike a number of its contemporaries, The Canadian Journal of Neurological Sciences is unique in its combination of clinical neurology, neurosurgery, child neurology and clinical neurophysiology, the four components of our Canadian Congress of Neurological Sciences.

We have a number of challenges ahead of us that include maintaining our leadership over newer journals of limited scope and making a smooth transition into the era of electronic publishing. For this we have important local expertise and you will be hearing more about our electronic initiatives in the near future.

In the last issue and in this issue, we honor several outstanding Canadian neuroscientists. This issue honors Dr. Charles Drake a Canadian neurosurgeon who revolutionized the treatment of vertebral-basilar territory cerebral aneurysms. We include an introductory "In Memoriam" by his colleagues, Gary Ferguson and Vladimir Hachinski; an address prepared by Dr.
Drake just before his death, on his early training years in Toronto; a reproduction of one of his famous "Track Sheets" used to record details of his aneurysm surgery; and a reproduction of one of Dr. Drake's articles originally published in the Journal of Neurosurgery. It is my hope that these articles in our Journal will help to highlight the outstanding contributions made by Dr. Drake to neurosurgery internationally.

In this issue we introduce a new educational article "Neuroimaging Highlight" edited by Drs. Mark Hudon and William Hu. Each subsequent issue of the Journal will include a Neuroimaging Highlight and I hope it will serve as an important feature for our readers.

This editor would like to acknowledge the superb editorial staff that will help me to guide the Journal, including Sally Gregg, Sue Impey and Margaret Peterson. I will continue to call on Canadian and international neuroscientists to help in our well established review process. In advance, I would like to thank those of you who can continue to contribute to our peer review.

Our Journal has international contributors and readership and the strength of its citations will depend on the quality of the work we can attract. We encourage Canadian and international authors to send us their best work.

Douglas Zochodne 